\title{
Targeted Next-Generation Sequencing Identifies Separate Causes of Hearing Loss in One Deaf Family and Variable Clinical Manifestations for the p.R161C Mutation in SOX10
}

\author{
Xiaoyu Yu $\mathbb{D}^{1,2,3}$ Yun Lin $\mathbb{D}^{1,2,3}$ and Hao $W u(\mathbb{D})^{1,2,3}$ \\ ${ }^{1}$ Department of Otorhinolaryngology-Head and Neck Surgery, Shanghai Ninth People's Hospital, Shanghai Jiao Tong University \\ School of Medicine, Shanghai, China \\ ${ }^{2}$ Ear Institute, Shanghai Jiao Tong University School of Medicine, Shanghai, China \\ ${ }^{3}$ Shanghai Key Laboratory of Translational Medicine on Ear and Nose Diseases, Shanghai, China
}

Correspondence should be addressed to Hao Wu; haowu@sh-jei.org

Received 22 April 2020; Revised 6 July 2020; Accepted 13 July 2020; Published 28 August 2020

Academic Editor: Renjie Chai

Copyright (c) 2020 Xiaoyu Yu et al. This is an open access article distributed under the Creative Commons Attribution License, which permits unrestricted use, distribution, and reproduction in any medium, provided the original work is properly cited.

Hearing loss is the most common sensory deficit in humans. Identifying the genetic cause and genotype-phenotype correlation of hearing loss is sometimes challenging due to extensive clinical and genetic heterogeneity. In this study, we applied targeted nextgeneration sequencing (NGS) to resolve the genetic etiology of hearing loss in a Chinese Han family with multiple affected family members. Targeted sequencing of 415 deafness-related genes identified the heterozygous c.481C $>\mathrm{T}$ (p.R161C) mutation in SOX10 and the homozygous c.235delC (p.L79Cfs*3) mutation in GJB2 as separate pathogenic mutations in distinct affected family members. The SOX10 c.481C > T (p.R161C) mutation has been previously reported in a Caucasian patient with Kallmann syndrome that features congenital hypogonadotropic hypogonadism with anosmia. In contrast, family members carrying the same p.R161C mutation in this study had variable Waardenburg syndrome-associated phenotypes (hearing loss and/or hair hypopigmentation) without olfactory or reproductive anomalies. Our results highlight the importance of applying comprehensive diagnostic approaches such as NGS in molecular diagnosis of hearing loss and show that the p.R161C mutation in SOX10 may be associated with a wide range of variable clinical manifestations.

\section{Introduction}

Hearing loss is the most prevalent neurosensory impairment in humans, affecting over half a billion people worldwide [1, 2]. In a mammals' inner ear, cochlear hair cells (HCs) take responsibility to convert the mechanical sound waves into electrical signals [3-5], which make the HCs very important for hearing function. Many previous reports have already shown that HCs can be injured due to genetic factors, noise, ototoxic drugs, aging, or inflammation [6-13]; and it is estimated that $50 \%-60 \%$ of early-onset hearing loss is due to genetic factors $[14,15]$. Based on the association with other clinical features, approximately $70 \%$ of genetic hearing loss is nonsyndromic and $30 \%$ is syndromic. Currently, more than 100 genes for nonsyndromic hearing loss have been identified, and over 700 different forms of syndromic hearing impairment have been described $[16,17]$. The extremely high genetic and phenotypic heterogeneity sometimes makes the diagnosis of genetic hearing loss challenging.

Mutations in SOX10 have been associated with various forms of syndromic hearing loss. SOX10 is a transcription factor involved in cell fate determination and cell lineage development, especially in the forming and differentiation of the neural crest [18]. A variety of mutations in SOX10 may result in various developmental defects including type II (WS2, OMIM 611584) and type IV (WS4, OMIM 613266) Waardenburg syndrome (WS) featuring auditory and pigmentary abnormalities, with the latter also exhibiting short-segment Hirschsprung disease (HD, OMIM 142623) [19-22]. Recently, mutations in SOX10 have been identified in a few patients with Kallmann syndrome (KS, OMIM 308700) with deafness, which is characterized by 
hypogonadotropic hypogonadism, anosmia and hearing loss [23-26]. However, it remains unknown if there is a specific genotype-phenotype correlation between certain SOX10 mutations and Kallmann syndrome.

In the present study, we applied targeted NGS to identify the genetic etiology of hearing loss in a moderate-sized Chinese Han family with apparently complex inheritance. In different affected family members, we identified separate genetic causes in recessive mutation in the GJB2 gene and dominant mutation in the SOX10 gene. Despite the fact that SOX10 c.481C>T (p.R161C) mutation was previously associated with Kallmann syndrome, family members with this mutation in our study had either normal or only WS2 (hearing loss and hair hypopigmentation) phenotype, indicating a rather variable clinical manifestation.

\section{Subjects and Methods}

2.1. Subjects and Clinical Assessments. The proband (Figure 1(a), IV-1) with bilateral profound sensorineural hearing loss was enrolled through the Department of Otorhinolaryngology at Shanghai Ninth People's Hospital. Four other subjects (II1-1, II1-2, II-1, and II-2) from the four-generation family and 100 Chinese Han normal hearing controls were also included in this study. The clinical evaluation included a detailed medical history questionnaire and a thorough physical examination. Auditory evaluations were performed in all participants, including otoscopic examination, otoacoustic emissions (OAEs), auditory evoked potentials (AEPs), or pure-tone audiometry. High-resolution computerized tomography (CT) scan of the temporal bone was performed in proband IV-1. Sense of smell was evaluated by self-report, questioning, or olfactory tests. Blood sample was collected from the proband and her family members, and total DNA was extracted from peripheral blood leukocytes using standard protocols.

2.2. Ethics Statement. Written informed consent was obtained from all study participants or their guardians. All experimental procedures in this study were approved by the Ethics Committee of Shanghai Ninth People's Hospital, Shanghai Jiao Tong University School of Medicine.

\subsection{Targeted Genomic Enrichment and Massively Parallel} Sequencing. The quality and quantity of genomic DNA were assessed by gel electrophoresis and spectrophotometry. Libraries were prepared using the Illumina standard protocol. Targeted enrichment of all exons and flanking splicing sites of 415 genes implicated in sensorineural hearing loss (for the list of genes, see Supplementary Table S1) was completed using MyGenostics Gencap ${ }^{\mathrm{TM}}$ capture kit (MyGenostics, Baltimore, $\mathrm{MD}$, USA) following the manufacturer's protocol. The enrichment libraries were sequenced on Illumina NextSeq 500 sequencer.

2.4. Bioinformatics Analysis. The high-quality reads were mapped to the human genome sequence (hg19) with a Burrows-Wheeler-Alignment Tool, and GATK HaplotypeCaller was used to detect small insertions or deletions (InDels) and Single Nucleotide Variants (SNVs) [27, 28]. The identified
SNVs and InDels were then annotated using the ANNOVAR software [29]. The missense, frameshift, nonsense, and splicing variants with a minor allele frequency $<1 \%$ were further interrogated as candidate pathogenic mutations. For allele frequencies, we used 1000 Genomes (http://www.1000genomes .org/), ESP6500 (http://evs.gs.washington.edu/EVS/), and ExAC (http://exac.broadinstitute.org/) databases. An ensemble tool REVEL (rare exome variant ensemble learner) was used to predict the pathogenicity of missense variants [30]. The ClinVar database and Human Gene Mutation Database (HGMD) were used to further annotate known pathogenic variants. Pathogenicity of the candidate variants was interpreted following American College of Medical Genetics and Genomics (ACMG) standards and guidelines 2015 [31].

2.5. Sanger Sequencing. The candidate variants in SOX10 and GJB2 gene were amplified by polymerase chain reaction (PCR) and analyzed by Sanger sequencing. The primer sequences for PCR amplification are provided in Supplementary Table S3.

\section{Results}

3.1. Clinical Findings. The female proband VI-1 was born from nonconsanguineous parents (Figure 1(a)). She failed the neonatal hearing screening, and further examination revealed bilateral profound sensorineural hearing loss (>95 dB, Figure 1(b)). Her computed tomography scan of the temporal bone revealed slight dilation of the posterior semicircular canals in both ears. When examined at 7 years of age, she had normal dark irides, normal fundus oculi, and no pigmentary alterations in the skin or hair. Dystopia canthorum, limb anomaly, and Hirschsprung disease were absent. Neurological examination was normal.

The proband's mother III-2 had prelingual, bilateral, profound hearing loss. Interview and visual inspection of III-2 did not find pigmentation defects and musculoskeletal anomalies. The father III-1 had normal hearing but reported to develop a white forelock at approximately 8 years of age and prematurely gray hair at 20 years of age. The paternal grandmother II-1 had severe congenital bilateral hearing loss and reported a frontal white forelock and premature graying of hair since approximately 15 years of age. All family members reported normal sense of smell, which was confirmed by olfactory tests. Both III-2 and II-1 had normal puberty and spontaneous pregnancy.

3.2. Genetic Analysis Results. Targeted next-generation sequencing of 415 deafness-related genes identified a homozygous c.235delC (p.L79Cfs *3) variant in GJB2 as the pathogenic cause of hearing loss for the mother III-2 (Figure 2(a)). In the proband IV -1 and her father III-1, we detected 10 and 12 rare (MAF $<0.01$ in public databases) heterogeneous nonsynonymous variants, respectively (Supplementary Table S2). Of these, the c.481C $>\mathrm{T}$ (p.R161C) variant in SOX10 has been previously reported resulting in Kallmann syndrome in a Caucasian patient [32]. It substitutes a wellconserved arginine by cystine in the high-mobility group (HMG) domain of SOX10 (Figure 2(b)). This variant was 


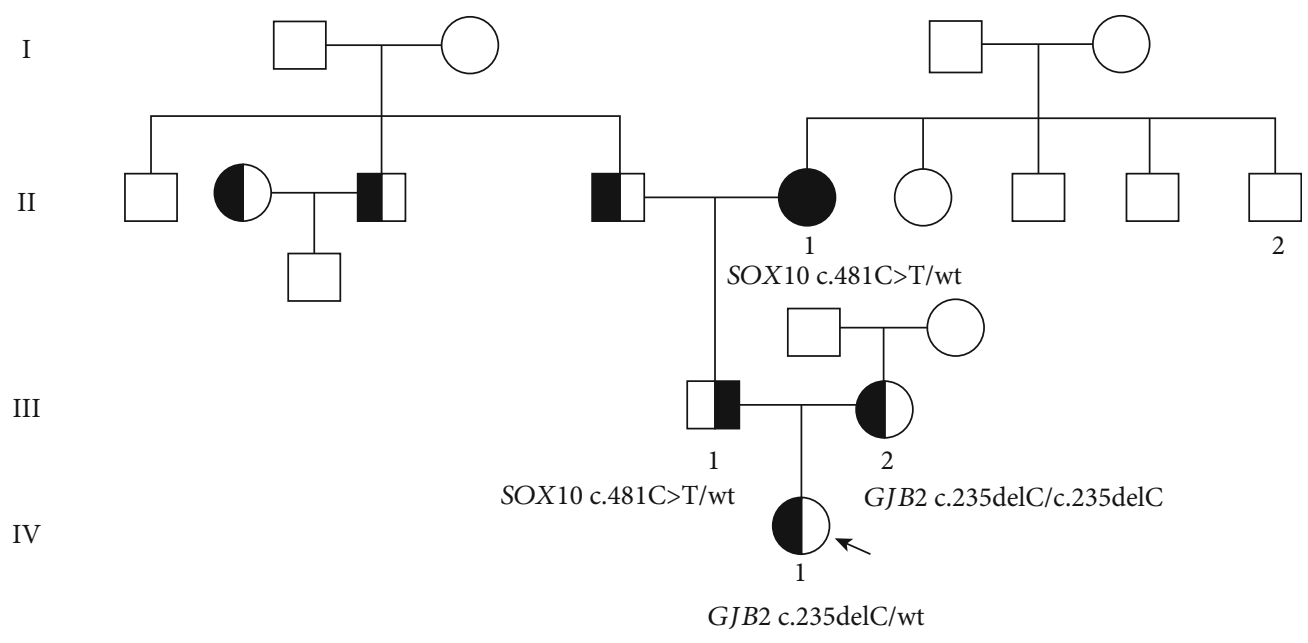

SOX 10 c. $481 \mathrm{C}>\mathrm{T} / \mathrm{wt}$

$\square \bigcirc$ Hearing loss
$\square$ Hair hypopigmentation

(a)

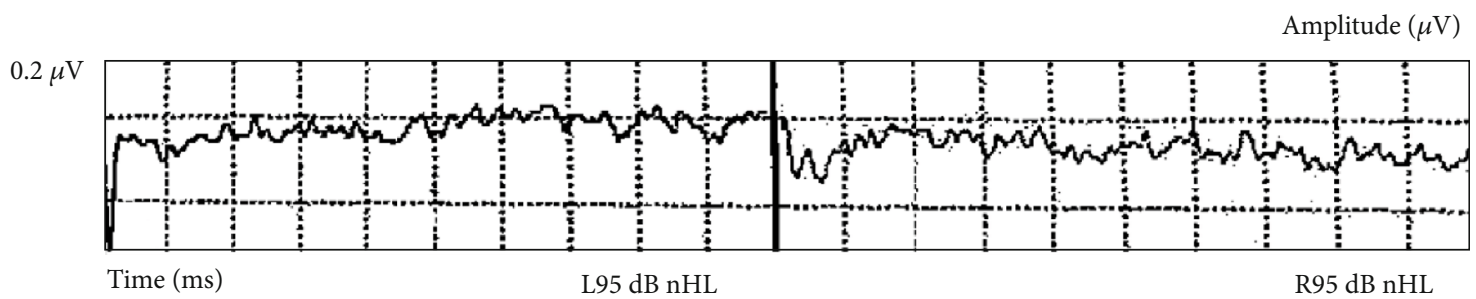

(b)

Figure 1: Pedigree of the family and clinical findings of the proband IV-1: (a) pedigree and genotype showing the c.481C>T (p.R161C) mutation in SOX10 and the c.235delC (p.L79Cfs*3) mutation in GJB2; (b) auditory evoked potentials showing bilateral profound sensorineural hearing loss.

not observed in genotyping of 100 Chinese Han normal controls and was predicted as damaging by in silico assessment with REVEL [30]. According to the 2015 ACMG guideline, c.481C>T (p.R161C) in SOX10 was classified as likely pathogenic (PS1+PM2). Sanger sequencing validated the presence of this variant in IV-1, III-1, and II-1, three individuals with WS-associated phenotypes (Figure 2(a)).

\section{Discussion}

The cause of hearing loss is extremely heterogeneous, and in many regions of the world, deaf people tend to marry with each other to form rather complex deaf families [33-40]. In one such family, we identified two separate genetic causes of hearing loss in distinct affected members, including the recessive c.235delC (p.L79Cfs $* 3$ ) mutation in GJB2 (III-2) and the dominant c.481C $>\mathrm{T}$ (p.R161C) mutation in SOX10 (II-1, III-1, and IV-1). While the c.235delC (p.L79Cfs $* 3$ ) mutation in GJB2 is quite common and well characterized in East Asians, the c.481C>T (p.R161C) mutation in SOX10 was far less frequent and its clinical manifestations were not consistent in different reports [32, 41].

In this study, the clinical manifestations of the family members carrying the c.481C $>\mathrm{T}$ (p.R161C) mutation in SOX10 are distinct along three different generations: typical WS2 phenotype (hearing loss and hair hypopigmentation) in the paternal grandmother, hair hypopigmentation only in the father, and hearing loss only in the proband. The c.481C > T (p.R161C) mutation affected the HMG domain of SOX10, which is the sequence-specific DNA-binding domain, and was predicted to be damaging by in silico assessment.

The same SOX10 c.481C > T (p.R161C) mutation has previously been reported in a Caucasian patient with Kallmann syndrome, in whom the presence or absence of hearing loss and pigmentation defect was not described (Table 1) [32]. Kallmann syndrome is a developmental disease that combines congenital hypogonadotropic hypogonadism with anosmia [42]. Our patients manifested hearing loss and hair hypopigmentation, but no anosmia or delayed puberty. This phenotypic difference suggests that other factors, such as modifier gene or epigenetic events, might contribute to the 
SOX 10 c. 481

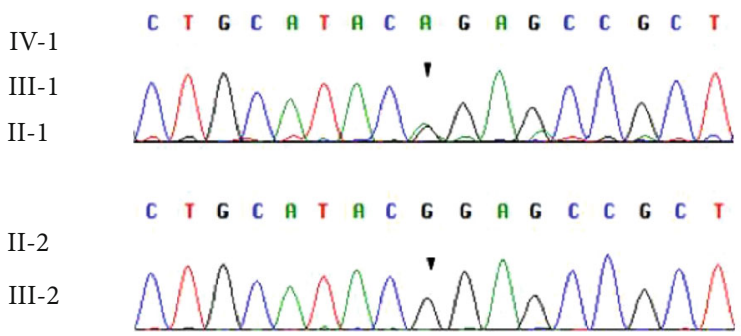

GJB2 c.235

III-2
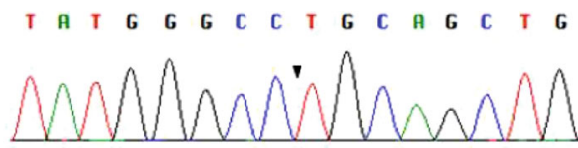

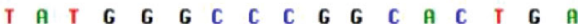

IV-1

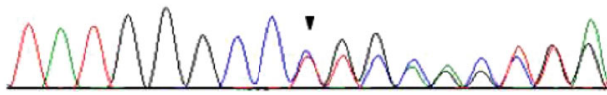

(a)

Home sapiens
Pan troglodytes
Rattus norvegicus
Gallus gallus
Danio rerio
Equus caballus
Xenopus tropicalis
Mus musculus

Home sapiens

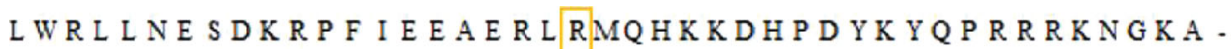
LWRLL NESDKRPFIEEAERLRMQHKKDHPD Y Y Y PRRRKNGKA. LWRLL N S DKR P I E A E R L RQHKKDHPDYK YQPRRRKNGKAL WRL L N S DKR P I E A E R RMQHKKDHPD YK Y PRRRKNGKA. LWRLL NE T D R P I E E A R L RKQHKKD Y P YK YQPRRRKNGKPG LWRLL NESDKR P I E E E R L RMQHKKDH D YK YQPRRRKNGKA -

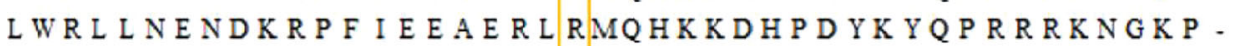
LWRLL NESDKRPFIEEAERLRMQHKKDHPDYKYQPRRRKNGA.

$\mathbf{\Lambda}$

(b)

FIgURE 2: The GJB2 c.235delC (p.L79Cfs*3) and the SOX10 c.481C>T (p.R161C) mutations identified in the family. (a) Sequence chromatogram showing the genotyping results of the family members. The proband IV-1, her father III-1, and the grandmother II-1 had heterogeneous SOX10 c.481C>T (p.R161C, arrow) mutation. The mother III-2 carried a homozygous GJB2 c.235delC (p.L79Cfs*3) mutation. (b) Alignment of SOX10 sequences from various species showing conservation of the arginine residue at position 161.

TABLE 1: Summary of clinical findings in patients with SOX10 c.481C>T (p.R161C) mutation.

\begin{tabular}{|c|c|c|c|c|c|}
\hline Patient & IV-1 & III-1 & II-1 & Marcos et al.[32] & Bademci et al.[41] \\
\hline$\overline{\text { Age }}$ & $7 y$ & $34 \mathrm{y}$ & $60 y$ & n.d. & $9 y ; 11 y$ \\
\hline Gender & $\mathrm{F}$ & M & $\mathrm{F}$ & n.d. & $\mathrm{F} ; \mathrm{M}$ \\
\hline \multicolumn{6}{|l|}{ Hearing loss/inner ear imaging } \\
\hline Hearing loss & Profound & - & Profound & n.d. & Profound \\
\hline Abnormal semicircular canal & Post. SCC dilatation & NA & NA & n.d. & n.d. \\
\hline \multicolumn{6}{|l|}{ Pigmentation defects } \\
\hline Pigmentary disturbances of iris & - & - & - & n.d. & n.d. \\
\hline Iris heterochromia & - & - & - & n.d. & n.d. \\
\hline Skin depigmentation & - & - & - & n.d. & n.d. \\
\hline White forelock & - & + & + & n.d. & n.d. \\
\hline Premature graying & - & + & + & n.d. & n.d. \\
\hline \multicolumn{6}{|l|}{ Eye anomalies } \\
\hline Telecanthus & - & - & - & n.d. & n.d. \\
\hline Retinal pigmentation defect & - & - & - & n.d. & n.d. \\
\hline \multicolumn{6}{|l|}{ Gastrointestine } \\
\hline Constipation & - & - & - & n.d. & n.d. \\
\hline Hirschsprung disease & - & - & - & n.d. & n.d. \\
\hline Hypogonadotropic hypogonadism & - & - & - & + & n.d. \\
\hline Delayed puberty & NA & - & - & + & n.d. \\
\hline Anosmia or severe hyposmia & - & - & - & + & n.d. \\
\hline \multicolumn{6}{|l|}{ Genetic } \\
\hline SOX10 mutation & c. $481 \mathrm{C}>\mathrm{T}$ & c. $481 \mathrm{C}>\mathrm{T}$ & c. $481 \mathrm{C}>\mathrm{T}$ & c. $481 \mathrm{C}>\mathrm{T}$ & c. $481 \mathrm{C}>\mathrm{T}$ \\
\hline
\end{tabular}

n.d.: not described; NA: not applicable; y: year. 


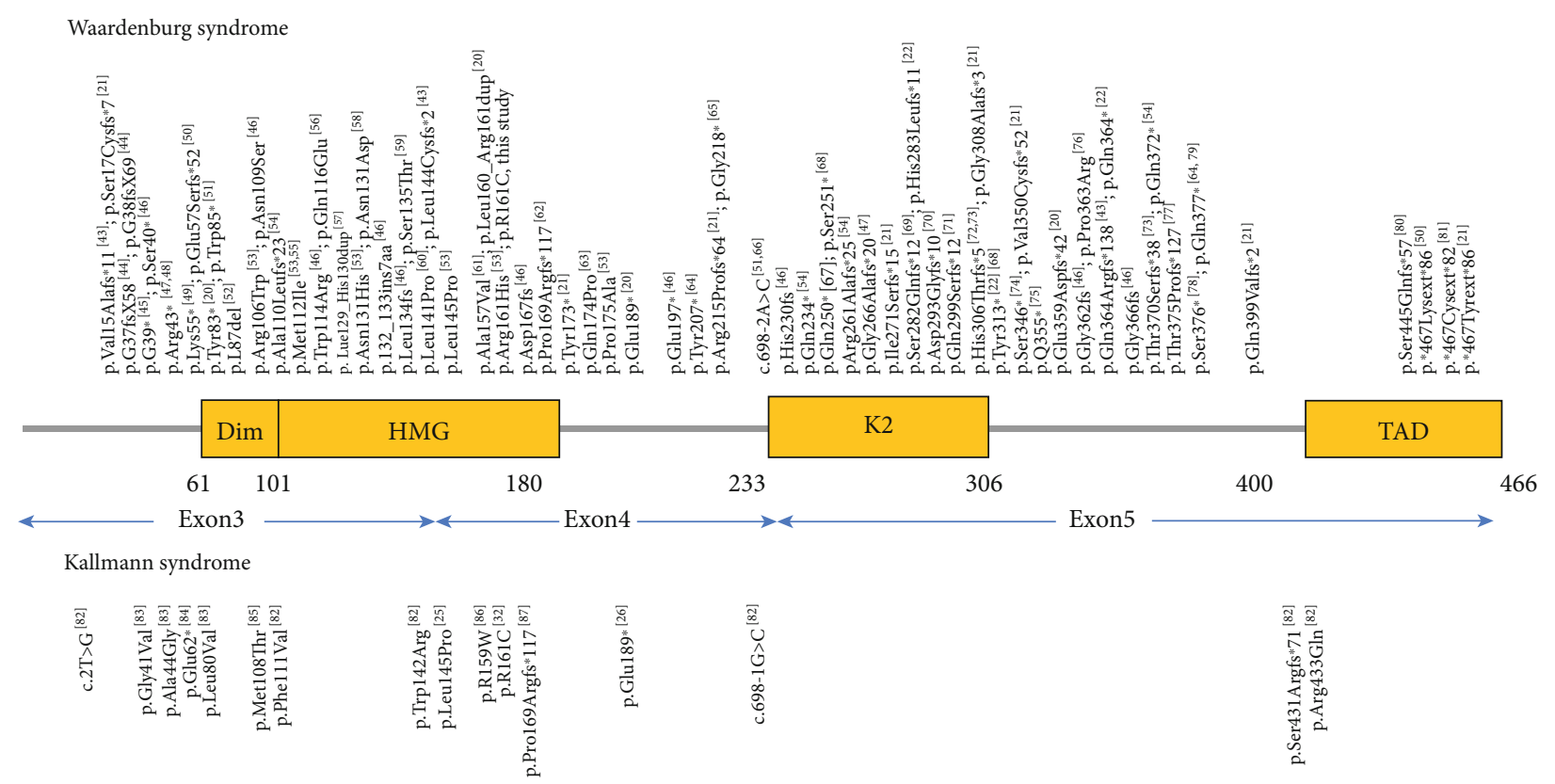

FIGURE 3: Schematic representation of the SOX10 domains and overview of SOX10 mutations and their associated phenotypes.

expression of the KS phenotypes. To date, the SOX10 c.481C $>\mathrm{T}$ (p.R161C) mutation has been identified in six patients from three families (Table 1) [32, 41]. Hearing loss was observed in four of the six patients and seems to be a consistent feature in mutation carriers, while hypogonadism and anosmia symptoms were described only in one patient.

Most of SOX10 mutations are private and were identified in sporadic cases, making it difficult to correlate the genotypes with the distinct disease phenotypes. Herein, we identified a KS-associated SOX10 mutation in a family with WS2, indicating that the same SOX10 mutation can underlie both WS and KS. Among over 80 published SOX10 mutations, three (p.Leu145Pro, p.Pro169Argfs $* 117$ and p.Glu189*) were also found to lead to different phenotypes (Figure 3) [43-87]. Further investigation is needed to clarify the underlying mechanisms of incomplete penetrance and high phenotypic variability caused by SOX10 mutations.

Our study also demonstrates that targeted NGS is a powerful strategy to discover causative genes in rare, heterogeneous disorders such as hearing loss. WS caused by SOX10 mutations can resemble nonsyndromic hearing loss in young children who do not present with pigmentary abnormality. Targeted NGS has the potential to identify such mutations which would improve the management of hearing loss by genetic counseling for the children and risk assessment of the relatives.

\section{Data Availability}

The data supporting the findings of this study are available within the article and the supplementary files.

\section{Conflicts of Interest}

The authors declare that they have no competing interests.

\section{Authors' Contributions}

$\mathrm{Hao} \mathrm{Wu}$ was responsible for the conceptualization, funding acquisition, project administration, supervision, writing, review, and editing. Xiaoyu Yu and Yun Lin were responsible for the formal analysis and investigation. Xiaoyu $\mathrm{Yu}$ was responsible for the writing of the original draft.

\section{Acknowledgments}

This research was supported by grants from the National Natural Science Foundation of China (81730028 to HW) and Shanghai Municipal Science and Technology Commission (14DZ2260300 to HW).

\section{Supplementary Materials}

Supplementary Table S1: a list of 415 deafness-related genes included in the targeted gene panel. Supplementary Table S2: candidate variants identified by targeted NGS in the proband VI-1 and her parents III-1 and III-2. Supplementary Table S3: primers used in this study. (Supplementary Materials)

\section{References}

[1] A. M. Sheffield and R. J. H. Smith, "The epidemiology of deafness," Cold Spring Harbor Perspectives in Medicine, vol. 9, no. 9, 2019.

[2] B. S. Wilson, D. L. Tucci, M. H. Merson, and G. M. O'Donoghue, "Global hearing health care: new findings and perspectives," Lancet, vol. 390, no. 10111, pp. 2503-2515, 2017.

[3] Y. Liu, J. Qi, X. Chen et al., "Critical role of spectrin in hearing development and deafness," Science Advances, vol. 5, no. 4, p. eaav7803, 2019. 
[4] Y. Wang, J. Li, X. Yao et al., "Loss of CIB2 causes profound hearing loss and abolishes mechanoelectrical transduction in mice," Frontiers in Molecular Neuroscience, vol. 10, p. 401, 2017.

[5] C. Zhu, C. Cheng, Y. Wang et al., "Loss of ARHGEF6 causes hair cell stereocilia deficits and hearing loss in mice," Frontiers in Molecular Neuroscience, vol. 11, p. 362, 2018.

[6] Z.-h. He, S.-y. Zou, M. Li et al., "The nuclear transcription factor FoxG1 affects the sensitivity of mimetic aging hair cells to inflammation by regulating autophagy pathways," Redox Biology, vol. 28, p. 101364, 2020.

[7] S. Gao, C. Cheng, M. Wang et al., "Blebbistatin inhibits neomycin-induced apoptosis in hair cell-like HEI-OC-1 cells and in cochlear hair cells," Frontiers in Cellular Neuroscience, vol. 13, 2020.

[8] Y. Zhang, W. Li, Z. He et al., "Pre-treatment with fasudil prevents neomycin-induced hair cell damage by reducing the accumulation of reactive oxygen species," Frontiers in Molecular Neuroscience, vol. 12, p. 264, 2019.

[9] W. Liu, X. Xu, Z. Fan et al., "Wnt signaling activates TP53induced glycolysis and apoptosis regulator and protects against cisplatin-induced spiral ganglion neuron damage in the mouse cochlea," Antioxidants \& Redox Signaling, vol. 30, no. 11, pp. 1389-1410, 2019.

[10] L. Liu, Y. Chen, J. Qi et al., "Wnt activation protects against neomycin-induced hair cell damage in the mouse cochlea," Cell Death \& Disease, vol. 7, no. 3, article e2136, 2016.

[11] H. Li, Y. Song, Z. He et al., "Meclofenamic acid reduces reactive oxygen species accumulation and apoptosis, inhibits excessive autophagy, and protects hair cell-like HEI-OC1 cells from cisplatin-induced damage," Frontiers in Cellular Neuroscience, vol. 12, p. 139, 2018.

[12] S. Zhang, Y. Zhang, Y. Dong et al., "Knockdown of Foxg1 in supporting cells increases the trans-differentiation of supporting cells into hair cells in the neonatal mouse cochlea," Cellular and Molecular Life Sciences : CMLS, vol. 77, no. 7, pp. 14011419, 2020.

[13] Z. He, L. Guo, Y. Shu et al., "Autophagy protects auditory hair cells against neomycin-induced damage," Autophagy, vol. 13, no. 11, pp. 1884-1904, 2017.

[14] W. E. Nance, “The genetics of deafness," Mental Retardation and Developmental Disabilities Research Reviews, vol. 9, no. 2, pp. 109-119, 2003.

[15] K. W. Chang, "Genetics of hearing loss-nonsyndromic," Otolaryngologic Clinics of North America, vol. 48, no. 6, pp. 10631072, 2015.

[16] R. J. Gorlin, R. J. Gorlin, H. V. Toriello, and M. M. Cohen, Hereditary Hearing Loss and Its Syndromes, Oxford University Press, 1995.

[17] T. Koffler, K. Ushakov, and K. B. Avraham, "Genetics of hearing loss: syndromic," Otolaryngologic Clinics of North America, vol. 48, no. 6, pp. 1041-1061, 2015.

[18] K. Kuhlbrodt, B. Herbarth, E. Sock, I. Hermans-Borgmeyer, and M. Wegner, "Sox10, a novel transcriptional modulator in glial cells," The Journal of neuroscience : the official journal of the Society for Neuroscience, vol. 18, no. 1, pp. 237250, 1998.

[19] N. Bondurand, F. Dastot-le Moal, L. Stanchina et al., "Deletions at the SOX10 gene locus cause Waardenburg syndrome types 2 and 4," American Journal of Human Genetics, vol. 81, no. 6 , pp. 1169-1185, 2007.
[20] V. Pingault, N. Bondurand, K. Kuhlbrodt et al., "SOX10 mutations in patients with Waardenburg-Hirschsprung disease," Nature Genetics, vol. 18, no. 2, pp. 171-173, 1998.

[21] V. Pingault, D. Ente, F. Dastot-Le Moal, M. Goossens, S. Marlin, and N. Bondurand, "Review and update of mutations causing Waardenburg syndrome," Human Mutation, vol. 31, no. 4, pp. 391-406, 2010.

[22] K. Inoue, M. Khajavi, T. Ohyama et al., "Molecular mechanism for distinct neurological phenotypes conveyed by allelic truncating mutations," Nature Genetics, vol. 36, no. 4, pp. 361369, 2004.

[23] A. K. Topaloglu and L. D. Kotan, "Genetics of hypogonadotropic hypogonadism," Endocrine development, vol. 29, pp. 36-49, 2016.

[24] P. Barraud, J. A. St John, C. C. Stolt, M. Wegner, and C. V. Baker, "Olfactory ensheathing glia are required for embryonic olfactory axon targeting and the migration of gonadotropinreleasing hormone neurons," Biology open, vol. 2, no. 7, pp. 750-759, 2013.

[25] E. Suzuki, Y. Izumi, Y. Chiba et al., "Loss-of-function SOX10 mutation in a patient with Kallmann syndrome, hearing loss, and iris hypopigmentation," Hormone Research in Pcediatrics, vol. 84, no. 3, pp. 212-216, 2015.

[26] F. Wang, S. Zhao, Y. Xie, W. Yang, and Z. Mo, "De novo SOX10 nonsense mutation in a patient with Kallmann syndrome, deafness, iris hypopigmentation, and hyperthyroidism," Annals of Clinical and Laboratory Science, vol. 48, no. 2, pp. 248-252, 2018.

[27] H. Li and R. Durbin, "Fast and accurate short read alignment with Burrows-Wheeler transform," Bioinformatics, vol. 25, no. 14, pp. 1754-1760, 2009.

[28] A. McKenna, M. Hanna, E. Banks et al., "The Genome Analysis Toolkit: a MapReduce framework for analyzing nextgeneration DNA sequencing data," Genome Research, vol. 20, no. 9, pp. 1297-1303, 2010.

[29] K. Wang, M. Li, and H. Hakonarson, “ANNOVAR: functional annotation of genetic variants from high-throughput sequencing data," Nucleic Acids Research, vol. 38, no. 16, article e164, 2010.

[30] N. M. Ioannidis, J. H. Rothstein, V. Pejaver et al., "REVEL: an ensemble method for predicting the pathogenicity of rare missense variants," American Journal of Human Genetics, vol. 99, no. 4, pp. 877-885, 2016.

[31] S. Richards, on behalf of the ACMG Laboratory Quality Assurance Committee, N. Aziz et al., "Standards and guidelines for the interpretation of sequence variants: a joint consensus recommendation of the American College of Medical Genetics and Genomics and the Association for Molecular Pathology," Genetics in Medicine, vol. 17, no. 5, pp. 405-423, 2015.

[32] S. Marcos, J. Sarfati, C. Leroy et al., "The prevalence of CHD7 missense versus truncating mutations is higher in patients with Kallmann syndrome than in typical CHARGE patients," The Journal of Clinical Endocrinology and Metabolism, vol. 99, no. 10, pp. E2138-E2143, 2014.

[33] S. Sun, M. Sun, Y. Zhang et al., "In vivo overexpression of Xlinked inhibitor of apoptosis protein protects against neomycin-induced hair cell loss in the apical turn of the cochlea during the ototoxic-sensitive period," Frontiers in Cellular Neuroscience, vol. 8, 2014.

[34] T. Wang, R. Chai, G. S. Kim et al., "Lgr5+ cells regenerate hair cells via proliferation and direct transdifferentiation in 
damaged neonatal mouse utricle," Nature Communications, vol. 6, no. 1, 2015.

[35] F. Tan, C. Chu, J. Qi et al., "AAV-ie enables safe and efficient gene transfer to inner ear cells," Nature Communications, vol. 10, no. 1, p. 3733, 2019.

[36] Z. He, et al.S. Sun, M. Waqas et al., "Reduced TRMU expression increases the sensitivity of hair-cell-like HEI-OC-1 cells to neomycin damage in vitro," Scientific Reports, vol. 6, no. 1, 2016.

[37] X. Yu, W. Liu, Z. Fan et al., "c-Myb knockdown increases the neomycin-induced damage to hair-cell-like HEI-OC1 cells in vitro," Scientific Reports, vol. 7, no. 1, 2017.

[38] Z. He, Q. Fang, H. Li et al., "The role of FOXG1 in the postnatal development and survival of mouse cochlear hair cells," Neuropharmacology, vol. 144, pp. 43-57, 2019.

[39] J. Qi, Y. Liu, C. Chu et al., “A cytoskeleton structure revealed by super-resolution fluorescence imaging in inner ear hair cells," Cell Discovery, vol. 5, no. 1, 2019.

[40] J. Qi, L. Zhang, F. Tan et al., "Espin distribution as revealed by super-resolution microscopy of stereocilia," American Journal of Translational Research, vol. 12, no. 1, pp. 130-141, 2020.

[41] G. Bademci, F. B. Cengiz, J. Foster II et al., "Variations in multiple syndromic deafness genes mimic non-syndromic hearing loss," Scientific Reports, vol. 6, no. 1, 2016.

[42] C. Dodé and J.-P. Hardelin, "Kallmann syndrome," European Journal of Human Genetics, vol. 17, no. 2, pp. 139-146, 2009.

[43] M. A. P. Bocángel, U. S. Melo, L. U. Alves et al., "Waardenburg syndrome: novel mutations in a large Brazilian sample," European Journal of Medical Genetics, vol. 61, no. 6, pp. 348-354, 2018.

[44] H. Chen, L. Jiang, Z. Xie et al., "Novel mutations of PAX3, MITF, and SOX10 genes in Chinese patients with type I or type II Waardenburg syndrome," Biochemical and Biophysical Research Communications, vol. 397, no. 1, pp. 70-74, 2010.

[45] Y. Arimoto, K. Namba, A. Nakano, and T. Matsunaga, "Chronic constipation recognized as a sign of a SOX10 mutation in a patient with Waardenburg syndrome," Gene, vol. 540, no. 2, pp. 258-262, 2014.

[46] L. Sun, X. Li, J. Shi et al., "Molecular etiology and genotypephenotype correlation of Chinese Han deaf patients with type I and type II Waardenburg syndrome," Scientific Reports, vol. 6, no. 1, 2016.

[47] V. Pingault, A. Guiochon-Mantel, N. Bondurand et al., "Peripheral neuropathy with hypomyelination, chronic intestinal pseudo-obstruction and deafness: a developmental "neural crest syndrome" related to a SOX10 mutation," Annals of Neurology, vol. 48, no. 4, pp. 671-676, 2000.

[48] J. Ma, Z. Zhang, H. C. Jiang et al., "A novel dominant mutation in the SOX10 gene in a Chinese family with Waardenburg syndrome type II," Molecular Medicine Reports, vol. 19, no. 3, pp. 1775-1780, 2019.

[49] J. Ma, T. S. Zhang, K. Lin et al., "Waardenburg syndrome type II in a Chinese patient caused by a novel nonsense mutation in the SOX10 gene," International Journal of Pediatric Otorhinolaryngology, vol. 85, pp. 56-61, 2016.

[50] M. H. Sham, V. C. Lui, B. L. Chen, M. Fu, and P. K. Tam, "Novel mutations of SOX10 suggest a dominant negative role in Waardenburg-Shah syndrome," Journal of Medical Genetics, vol. 38, no. 9, article E30, pp. 30e-330, 2001.

[51] L. Jiang, H. Chen, W. Jiang et al., "Novel mutations in the SOX10 gene in the first two Chinese cases of type IV Waarden- burg syndrome," Biochemical and Biophysical Research Communications, vol. 408, no. 4, pp. 620-624, 2011.

[52] K. Chen, L. Zong, M. Liu et al., "De novo dominant mutation of SOX10 gene in a Chinese family with Waardenburg syndrome type II," International Journal of Pediatric Otorhinolaryngology, vol. 78, no. 6, pp. 926-929, 2014.

[53] A. Chaoui, Y. Watanabe, R. Touraine et al., "Identification and functional analysis of SOX10 missense mutations in different subtypes of Waardenburg syndrome," Human Mutation, vol. 32, no. 12, pp. 1436-1449, 2011.

[54] V. Pingault, M. Girard, N. Bondurand et al., "SOX10 mutations in chronic intestinal pseudo-obstruction suggest a complex physiopathological mechanism," Human Genetics, vol. 111, no. 2, pp. 198-206, 2002.

[55] V. Pingault, L. Pierre-Louis, A. Chaoui et al., "Phenotypic similarities and differences in patients with a p.Met112Ile mutation in SOX10," American Journal of Medical Genetics Part A, vol. 164a, no. 9, pp. 2344-2350, 2014.

[56] P. Bogdanova-Mihaylova, M. D. Alexander, R. P. J. Murphy, and S. M. Murphy, "Waardenburg syndrome: a rare cause of inherited neuropathy due to SOX10 mutation," Journal of the Peripheral Nervous System: JPNS, vol. 22, no. 3, pp. 219-223, 2017.

[57] A. Hemmi, K. Okamura, R. Tazawa et al., "Waardenburg syndrome type IIE in a Japanese patient caused by a novel nonframe-shift duplication mutation in the SOX10 gene," The Journal of Dermatology, vol. 45, no. 5, pp. e110-e111, 2018.

[58] K. Okamura, N. Oiso, G. Tamiya et al., "Waardenburg syndrome type IIE in a Japanese patient caused by a novel missense mutation in the SOX10 gene," The Journal of Dermatology, vol. 42, no. 12, pp. 1211-1212, 2015.

[59] N. Bondurand, K. Kuhlbrodt, V. Pingault et al., "A molecular analysis of the Yemenite deaf-blind hypopigmentation syndrome: SOX10 dysfunction causes different neurocristopathies," Human Molecular Genetics, vol. 8, no. 9, pp. 17851789, 1999.

[60] N. Jalilian, M. A. Tabatabaiefar, H. Alimadadi, and M. R. Noori-Daloii, "SOX10 mutation causes Waardenburg syndrome associated with distinctive phenotypic features in an Iranian family: a clue for phenotype-directed genetic analysis," International Journal of Pediatric Otorhinolaryngology, vol. 96, pp. 122-126, 2017.

[61] M. Morín, A. Viñuela, T. Rivera et al., "A de novo missense mutation in the gene encoding the SOX10 transcription factor in a Spanish sporadic case of Waardenburg syndrome type IV," American Journal of Medical Genetics Part A, vol. 146a, no. 8, pp. 1032-1037, 2008.

[62] M. Iso, M. Fukami, R. Horikawa, N. Azuma, N. Kawashiro, and T. Ogata, "SOX10 mutation in Waardenburg syndrome type II," American Journal of Medical Genetics Part A, vol. 146a, no. 16, pp. 2162-2163, 2008.

[63] C. P. Barnett, R. Mendoza-Londono, S. Blaser et al., “Aplasia of cochlear nerves and olfactory bulbs in association with SOX10 mutation," American Journal of Medical Genetics Part A, vol. 149a, no. 3, pp. 431-436, 2009.

[64] E. M. Southard-Smith, M. Angrist, J. S. Ellison et al., "The Sox10(Dom) mouse: modeling the genetic variation of Waardenburg-Shah (WS4) syndrome," Genome Research, vol. 9, no. 3, pp. 215-225, 1999.

[65] N. Suzuki, H. Mutai, F. Miya et al., “A case report of reversible generalized seizures in a patient with Waardenburg syndrome 
associated with a novel nonsense mutation in the penultimate exon of SOX10," BMC Pediatrics, vol. 18, no. 1, p. 171, 2018.

[66] Y. Sznajer, C. Coldéa, F. Meire, I. Delpierre, T. Sekhara, and R. L. Touraine, "A de novo SOX10 mutation causing severe type 4 Waardenburg syndrome without Hirschsprung disease," American Journal of Medical Genetics Part A, vol. 146a, no. 8, pp. 1038-1041, 2008.

[67] K. Inoue, K. Shilo, C. F. Boerkoel et al., "Congenital hypomyelinating neuropathy, central dysmyelination, and Waardenburg-Hirschsprung disease: phenotypes linked by SOX10 mutation," Annals of Neurology, vol. 52, no. 6, pp. 836-842, 2002.

[68] R. L. Touraine, T. Attié-Bitach, E. Manceau et al., "Neurological phenotype in Waardenburg syndrome type 4 correlates with novel SOX10 truncating mutations and expression in developing brain," American Journal of Human Genetics, vol. 66, no. 5, pp. 1496-1503, 2000.

[69] Y. Akutsu, K. Shirai, A. Takei et al., "A patient with peripheral demyelinating neuropathy, central dysmyelinating leukodystrophy, Waardenburg syndrome, and severe hypoganglionosis associated with a novel SOX10 mutation," American Journal of Medical Genetics Part A, vol. 176, no. 5, pp. 1195-1199, 2018.

[70] A. Unzicker, V. Pingault, T. Meyer, S. Rauthe, A. Schütz, and S. Kunzmann, "A novel SOX10 mutation in a patient with PCWH who developed hypoxic-ischemic encephalopathy after E. coli sepsis," European Journal of Pediatrics, vol. 170, no. 11, pp. 1475-1480, 2011.

[71] A. R. Hogan, K. A. Rao, W. L. Thorson, H. L. Neville, J. E. Sola, and E. A. Perez, "Waardenburg syndrome type IV de novo SOX10 variant causing chronic intestinal pseudo-obstruction," Pediatric Gastroenterology, Hepatology \& Nutrition, vol. 22, no. 5, pp. 487-492, 2019.

[72] A. Viñuela, M. Morín, M. Villamar et al., "Genetic and phenotypic heterogeneity in two novel cases of Waardenburg syndrome type IV," American Journal of Medical Genetics Part A, vol. 149a, no. 10, pp. 2296-2302, 2009.

[73] R. M. Fernández, R. Núñez-Ramos, M. V. Enguix-Riego et al., "Waardenburg syndrome type 4: report of two new cases caused by SOX10 mutations in Spain," American Journal of Medical Genetics Part A, vol. 164a, no. 2, pp. 542-547, 2014.

[74] J. B. G. M. Verheij, D. A. Sival, J. H. van der Hoeven et al., "Shah-Waardenburg syndrome and PCWH associated with SOX10 mutations: a case report and review of the literature," European Journal of Paediatric Neurology, vol. 10, no. 1, pp. 11-17, 2006.

[75] H. H. Wang, H. S. Chen, H. B. Li et al., "Identification and functional analysis of a novel mutation in the SOX10 gene associated with Waardenburg syndrome type IV," Gene, vol. 538, no. 1, pp. 36-41, 2014.

[76] H. J. Jung, S. A. Jin, S. J. N. Choi, S. C. Lee, and S. J. Yun, “A de novo SOX10 mutation in a patient with Waardenburg syndrome type IV," Journal of the American Academy of Dermatology, vol. 68, no. 6, pp. e177-e178, 2013.

[77] S. B. Minami, K. Nara, H. Mutai et al., "A clinical and genetic study of 16 Japanese families with Waardenburg syndrome," Gene, vol. 704, pp. 86-90, 2019.

[78] F. Toki, N. Suzuki, K. Inoue et al., "Intestinal aganglionosis associated with the Waardenburg syndrome: report of two cases and review of the literature," Pediatric Surgery International, vol. 19, no. 11, pp. 725-728, 2003.
[79] T. Oshimo, K. Fukai, Y. Abe et al., "Pediatric case report: clinical profile of a patient with PCWH with p.Q377X nonsense mutation in the SOX10 gene," The Journal of Dermatology, vol. 39, no. 12, pp. 1022-1025, 2012.

[80] X. Wang, Y. Zhu, N. Shen et al., "A de novo deletion mutation in SOX10 in a Chinese family with Waardenburg syndrome type 4," Scientific Reports, vol. 7, no. 1, 2017.

[81] K. Inoue, Y. Tanabe, and J. R. Lupski, "Myelin deficiencies in both the central and the peripheral nervous systems associated with a SOX10 mutation," Annals of Neurology, vol. 46, no. 3, pp. 313-318, 1999.

[82] V. Pingault, V. Bodereau, V. Baral et al., "Loss-of-function mutations in SOX10 cause Kallmann syndrome with deafness," American Journal of Human Genetics, vol. 92, no. 5, pp. 707-724, 2013.

[83] W. Dai, J. Wu, Y. Zhao et al., "Functional analysis of SOX10 mutations identified in Chinese patients with Kallmann syndrome," Gene, vol. 702, pp. 99-106, 2019.

[84] K. Vaaralahti, J. Tommiska, V. Tillmann et al., "De novo SOX10 nonsense mutation in a patient with Kallmann syndrome and hearing loss," Pediatric Research, vol. 76, no. 1, pp. 115-116, 2014.

[85] L. Maione, S. Brailly-Tabard, J. Nevoux, J. Bouligand, and J. Young, "Reversal of congenital hypogonadotropic hypogonadism in a man with Kallmann syndrome due to SOX10 mutation," Clinical Endocrinology, vol. 85, no. 6, pp. 988-989, 2016.

[86] Q. Zhang, H. H. He, M. U. Janjua et al., "Identification of two novel mutations in three Chinese families with Kallmann syndrome using whole exome sequencing," Andrologia, vol. 52, no. 7, article e13594, 2020.

[87] Y. Izumi, I. Musha, E. Suzuki et al., "Hypogonadotropic hypogonadism in a female patient previously diagnosed as having Waardenburg syndrome due to a sox10 mutation," Endocrine, vol. 49, no. 2, pp. 553-556, 2015. 